\title{
EVALUACIÓN DEL RENDIMIENTO DE VINAGRE OBTENIDO DE LA PULPA DE BANANO (MUSA PARADISIACA) MEDIANTE FERMENTACIÓN BIFÁSICA ALCOHÓLICA Y ACÉTICA
}

\section{EVALUATION OF THE VINEGAR PERFORMANCE OBTAINED FROM THE BANANA PULP (MUSA PARADISIACA) THROUGH THE ALCOHOLIC AND ACETIC BIFASSIC FERMENTATION}

\author{
Intriago-Ponce María ${ }^{1 *}$; López-Zambrano Lisbeth²; Loor-García Claudio³ Mayorga- \\ Zambrano Jefferson ${ }^{4}$; Zambrano-Zambrano Gabriel ${ }^{5}$ \\ 1,2,3,4,5Estudiantes de Ingeniería Química, Facultad de Ciencias Matemáticas, Físicas y \\ Químicas, Universidad Técnica de Manabí. Área de Tecnología, Programa de \\ Ingeniería Química, Ingeniería de los Bioprocesos. Portoviejo, Ecuador.
}

*Correo: mintriago3724@utm.edu.ec

\begin{abstract}
Resumen
La importación de vinagre y sucedáneos del vinagre en Ecuador es constante. El vinagre es un líquido miscible, con sabor agrio, proveniente de la fermentación acética del vino a partir, inicialmente, de azúcares fermentables. El objetivo del presente proyecto consistió en evaluar el rendimiento de vinagre obtenido de la pulpa de banano (musa paradisiaca) mediante la fermentación bifásica alcohólica y acética; así como también estimar la producción a una escala industrial junto con el diseño de los reactores para el proceso. Los resultados obtenidos mostraron que inicialmente la producción de etanol se mantiene estable durante la mayor parte del proceso, lo cual brinda un rendimiento del $75 \%$. Posteriormente en la fermentación acética se obtiene un rendimiento del $16,1 \%$. Finalmente se concluye que para el caso de la fermentación acética es preciso el control de cultivo y crecimiento con el respectivo ajuste de variables para obtener así mayores rendimientos, también es necesaria la implementación de biorreactores en serie como criterio que garantice el mejor desempeño del proceso productivo.
\end{abstract}

Palabras clave: banano, fermentación bifásica, vinagre, biorreactores.

\begin{abstract}
The import of vinegar and substitutes for vinegar in Ecuador is constant. Vinegar is a miscible liquid, with a sour taste, coming from the acetic fermentation of wine, initially, from fermentable sugars. The objective of this project was to evaluate the yield of vinegar obtained from the banana pulp (musa paradisiaca) through the biphasic alcoholic and acetic fermentation as well as estimate the production on an industrial scale together with the design of the reactors for the process. The results obtained showed that initially ethanol production remains stable during most of the process, which yields a yield of $75 \%$. Subsequently in the acetic fermentation a yield of $16.1 \%$ is obtained. Finally, it is concluded that in the case of acetic fermentation, the control of culture and growth is necessary throughout the process with the respective adjustment of temperature, mixing and oxygenation variables to obtain higher yields, the implementation of bioreactors in series as a criterion that guarantees the best performance of the production process.
\end{abstract}

Keywords: banana, biphasic fermentation, vinegar, bioreactor.

Información del manuscrito:

Fecha de recepción: 03 de mayo de 2019

Fecha de aceptación: 27 de junio de 2019

Fecha de publicación: 10 de julio de 2019 


\section{Introducción}

El proceso biotecnológico para la obtención de vinagre consiste en una oxidación biológica mediante dos reacciones bioquímicas: alcohólica y acética, en la que el sustrato se oxida parcialmente por medio de bacterias para producir ácido acético (Sellmer-Wilsberg, 2016); el resultado de la transformación del etanol contenido en el medio inicial es una solución llamada vinagre (Llaguno \& Polo, 1991). Para obtener el ácido acético de una fruta deben existir algunas condiciones, tales como temperatura, $\mathrm{pH}$, grados de acidez y oxigenación, previo a ello debe ocurrir la fermentación alcohólica, ésta es conducida generalmente por las levaduras que pertenecen al género Saccharomyces y a la especie cerevisiae (Mesas \& Alegre, 1999); industrialmente estas levaduras son organismos altamente especializados debido a su gran utilidad (Querol et al., 2003). Las levaduras fermentativas necesitan los azúcares para su catabolismo; es decir, para obtener la energía necesaria para sus procesos vitales, pero además necesitan otros substratos para su óptimo desarrollo y rendimiento; naturalmente se añaden micro nutrientes y fuentes de nitrógeno como fosfato diamónico, cloruro amónico, sulfato amónico o carbonato amónico, en concentraciones de 0,1-0,3 g.L-1 (Mallouchos, 2003).

La segunda etapa es la fermentación acética, que se concede con la finalización del proceso de la fermentación alcohólica y se puede determinar por la interrupción de producción del alcohol etílico, en esta etapa comienza a formarse ácido acético en un medio estrictamente aerobio y ácido; y por tanto, intervienen bacterias Acetobacter aceti (Tesfaye, 2002). Estudios de obtención de vinagre recomiendan la adición de una solución de minerales y fuente nitrogenada de la siguiente composición: 0,8 g.L-1 de $\mathrm{KH}_{2} \mathrm{PO}_{4}$; 0,35 g. $\mathrm{L}^{-1}$ de $\mathrm{KH}_{2} \mathrm{PO}_{4}$. $3 \mathrm{H}_{2} \mathrm{O} ; 1,5 \mathrm{~g}$. $\mathrm{L}^{-1}$ de $(\mathrm{NH} 4)_{2} \mathrm{SO}_{4}$ con el objetivo de aumentar rendimientos hasta un 30\% (Ferreyra \& Schvab, 2014)

La incorporación de aire es un proceso esencial, dado el carácter aerobio de las bacterias acéticas, además de la cantidad de aire suministrado se debe considerar la pureza y calidad de éste, ya que las bacterias acéticas son sensibles a 
contaminantes presentes en el aire.

Según Peláez et al, (2011) en este tipo de fermentaciones, la concentración de oxígeno disuelto es uno de los factores determinantes de la velocidad global del proceso; sin embargo, cuando se aborda el modelado cinético de la fermentación acética, se suele evitar la presencia de parámetros relacionados con el oxígeno, de forma que no se han propuesto modelos que incluyan dicha variable con carácter cuantitativo en las ecuaciones cinéticas.

El banano tiene un considerable valor nutricional y son conocidos por su alto contenido en carbohidratos, potasio y fósforo (ICBF, 2005). Por cada $100 \mathrm{~g}$ de banano se tiene:

\section{Tabla 1.}

Composición de azúcares en el banano

\begin{tabular}{|lc|}
\hline Nutrientes & Valor \\
\hline \hline Azucares totales & $12.23 \mathrm{~g}$ \\
Sacarosa & $2.39 \mathrm{~g}$ \\
Glucosa & $4.98 \mathrm{~g}$ \\
Fructosa & $4.85 \mathrm{~g}$ \\
Lactosa & $0,0 \mathrm{~g}$ \\
Maltosa & $0,01 \mathrm{~g}$ \\
Galactosa & $0,0 \mathrm{~g}$ \\
\hline
\end{tabular}

Fuente: Romero, 2002.

El objetivo del presente artículo es evaluar el rendimiento de vinagre obtenido de la pulpa de banano mediante la fermentación bifásica alcohólica y acética, así como también estimar la producción a una escala industrial junto con el diseño de los reactores para el proceso.

\section{Materiales y métodos}

El sustrato fue adquirido en el mercado municipal de la ciudad de Portoviejo, Ecuador. Como tratamiento previo a la fermentación, el sustrato es sometido a un proceso de licuado para disminuir el tamaño de la partícula y así obtener una pasta que posteriormente se diluye con agua en una relación 2:1 (Blanco et al., 2012). Se evaluaron los grados Brix ( ${ }^{\circ}$ Brix) del sustrato mediante un refractómetro portátil graduado de 0 $-32 \%$ que ofrece una exactitud de $0,2 \%$ y se utilizó el método gravimétrico (peso seco) para la identificación del peso inicial del sustrato, ya que puede estar compuesto de material inerte, polímeros extracelulares y materia orgánica adsorbida que interfiere con el dato exacto de la biomasa generada durante la experimentación (Arnáiz et al., 2000).

La concentración recomendada de Saccharomyces cerevisae fue de 0,4 g.L ${ }^{-1}$ de sustrato. Se preparó un 
volumen de $3 \mathrm{~L}$ de mosto y durante 5 días se evaluó el crecimiento del microorganismo, el consumo del sustrato y la formación del producto.

La levadura empleada se activó con azúcar y temperatura $\left(35^{\circ} \mathrm{C}\right)$, durante 15 minutos (Barre \& Stalin, 2003).

Para cuantificar la producción de $\mathrm{CO}_{2}$ se empleó $\mathrm{NaOH}$ como reactivo adsorbente, en una disolución al 40\% P/V (Yank et al., 2015). El producto se evalúa acorde al método de análisis INEN 0360 para grados de alcohol (INEN, 2013). Posterior a la fermentación alcohólica se filtra y se verte el producto líquido a otro recipiente donde se llevó a cabo la fermentación acética. Se inoculó con $10 \mathrm{~mL}$ de cultivo madre de Acetobacter aceti, la cantidad de aire suministrada es de 27,8 L.d $^{-1}$ calculada mediante balances estequiométricos de los requerimientos teóricos más el $90 \%$ por efectos de solubilidad del oxígeno (Escalante, 2011). La acidez total fue evaluada mediante el método de análisis INEN 0341 (INEN, 2012).

\subsection{Obtención del cultivo madre de Acetobacter aceti}

Se dispuso el sustrato en un recipiente con amplia superficie de contacto para la oxigenación, tapado con gasas para evitar la contaminación física del cultivo. El proceso consistió en dejar en reposo el banano, para que al cabo de aproximadamente 6 días, las bacterias acéticas creen una película de celulosa en la superficie. A través del incremento diario de la acidez del medio se puede constatar que existe la presencia de bacterias del género acetobacter, sin embargo, se corroboró la presencia mediante la tinción de gram (Hurtado, 2011). Desde el primer día se alimentó con sustrato, ya que el grado de alcohol allí es de $2^{\circ} \mathrm{GL}$, se añadió $1 \mathrm{~mL}$ de etanol al $94 \%$ para mantener vivas las bacterias.

\section{Resultados y discusión}

El sustrato posee un ${ }^{\circ}$ Brix de $10,5 \%$. Los resultados obtenidos de la fermentación alcohólica se muestran a continuación en las figuras 1 y 2 . 


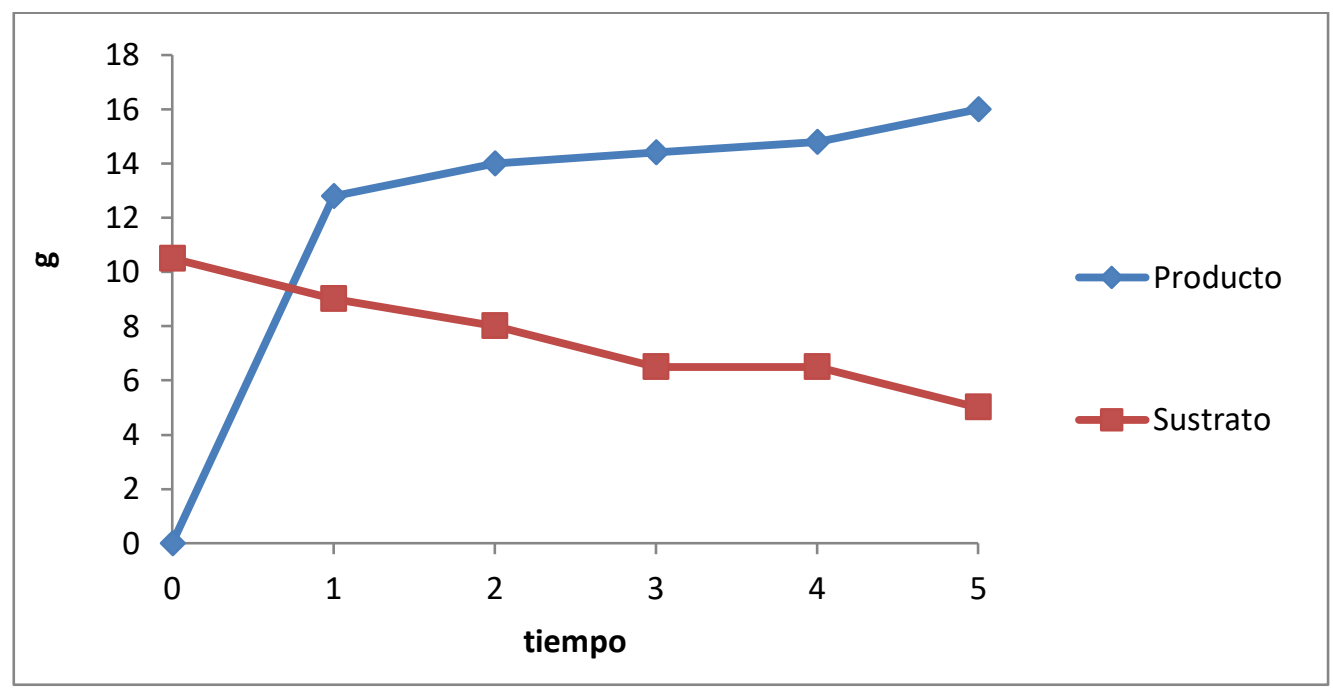

Figura 1. Cinética de consumo de sacarosa y formación de etanol

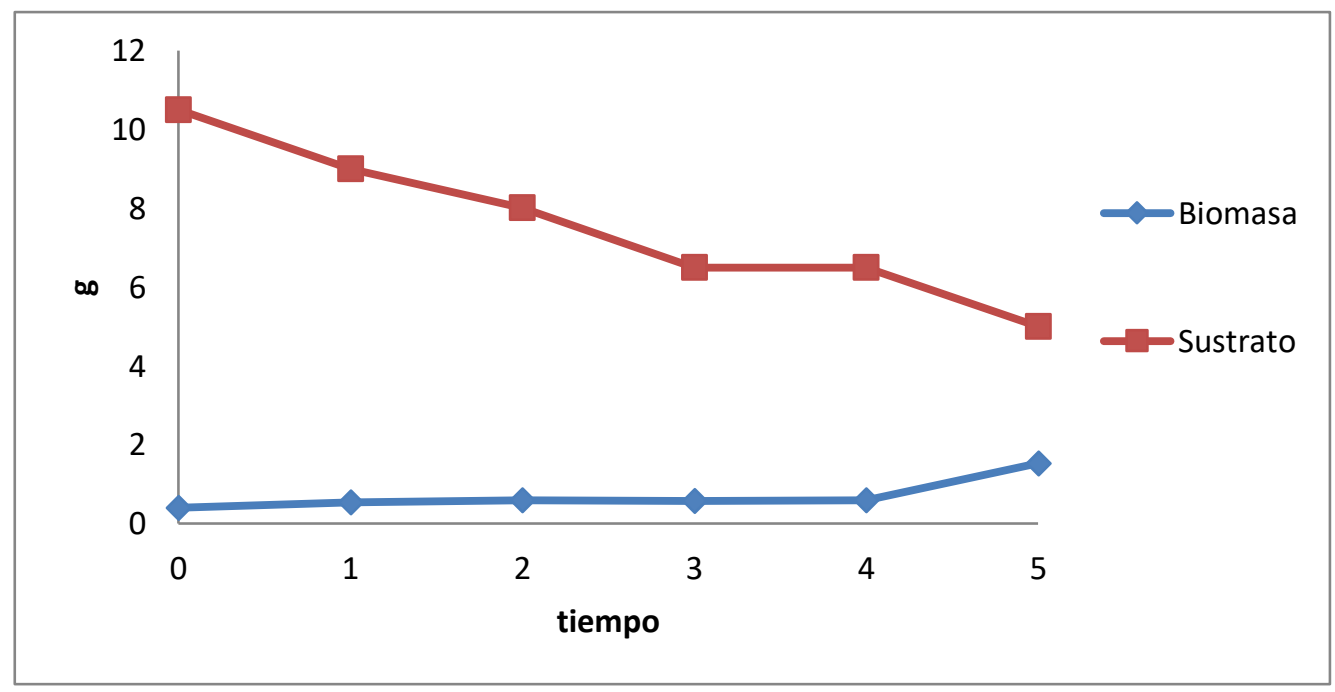

Figura 2. Cinética de consumo de sacarosa y generación de Saccharomyces cerevisae.

Experimentalmente se obtiene que por cada gramo de sacarosa se producen $0,2051 \mathrm{~g}$ de biomasa. En función de lo observado se refleja en la tabla 2 los coeficientes de la reacción obtenidos mediante balance estequiométrico.
Tabla 2.

Coeficientes estequiométricos

\begin{tabular}{|ccc|}
\hline Coeficiente & Moles & Gramos \\
\hline \hline a & 0,0029 & 1 \\
b & 0,0573 & 0,0283 \\
c & 2,8515 & 0,2051 \\
d & 3,1447 & 0,4043 \\
e & 0,2834 & 0,0149 \\
f & 3,0019 & 0,4040 \\
\hline
\end{tabular}


Tabla 3.

Comparación de los rendimientos

\begin{tabular}{|lc|}
\hline Rendimiento & Producto/Sustrato \\
\hline \hline Experimental & 0,4040 \\
Teórico & 0,5382 \\
\%Rendimiento & 75 \\
\hline
\end{tabular}

El sustrato posee 20 GL. Los resultados obtenidos de la fermentación acética se muestran a continuación en las figuras 3 y 4 .

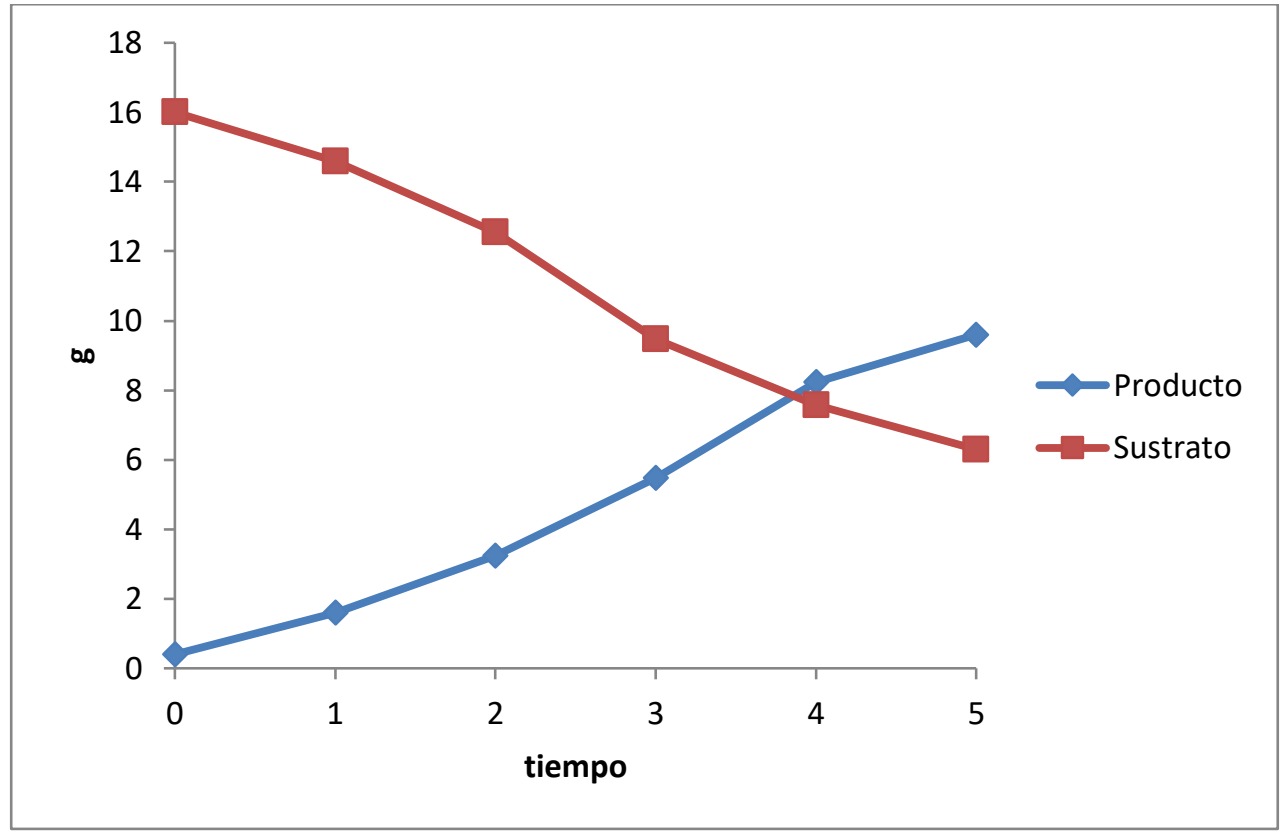

Figura 3. Cinética de consumo de etanol y formación de ácido acético

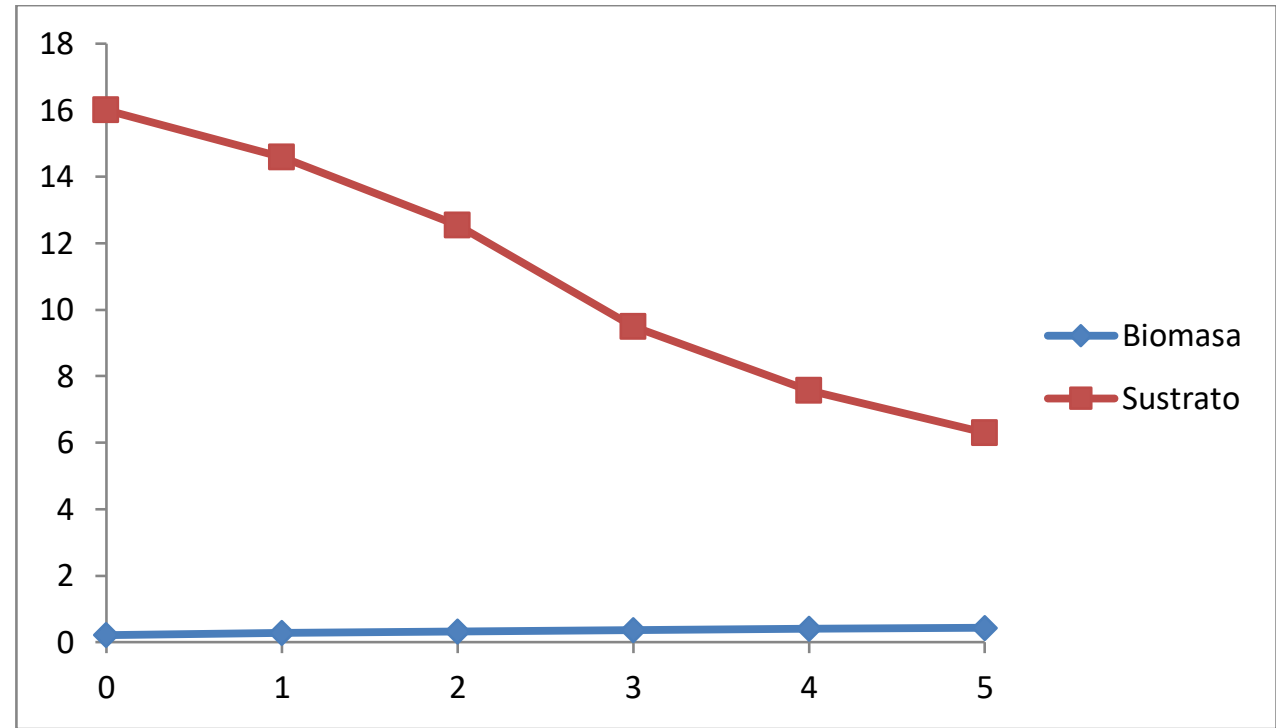

Figura 4. Cinética de consumo de etanol y generación de Acetobacter 
Experimentalmente se obtiene que por cada gramo de etanol se producen $0,2750 \mathrm{~g}$ de biomasa y $1,054 \mathrm{~g}$ de ácido acético.

Tabla 4.

Coeficientes estequiométricos de la fermentación acética

\begin{tabular}{|ccc|}
\hline Coeficiente & Moles & Gramos \\
\hline \hline $\mathrm{x}$ & 0,0217 & 1 \\
$\mathrm{a}$ & 1,4980 & 1,171 \\
$\mathrm{~b}$ & 0,0092 & 0,0034 \\
$\mathrm{c}$ & 2,8515 & 0,0228 \\
$\mathrm{~d}$ & 0,4990 & 0,4760 \\
$\mathrm{e}$ & 1,5270 & 0,5960 \\
$\mathrm{f}$ & 3,0019 & 0,9480 \\
\hline
\end{tabular}

Tabla 5.

Comparación de los rendimientos obtenidos

\begin{tabular}{|lc|}
\hline Rendimiento & Producto/Sustrato \\
\hline \hline Experimental & 0,9480 \\
Teórico & 5,8650 \\
\%Rendimiento & 16,1 \\
\hline
\end{tabular}

Para el diseño de los reactores se plantea el escenario de producción a escala industrial, para ello se determinó bibliográficamente la demanda potencial de consumo de vinagre en Manabí, la cual se establece en 1195,02 L.d-1 (CFN, 2017).

Tabla 6.

Balance de masa en fermentación alcohólica, en Kg

\begin{tabular}{|lcccc|}
\hline Componente & Ingresa & Consume & Forma & Sale \\
\hline \hline $\mathrm{C}_{12} \mathrm{H}_{22} \mathrm{O}_{11}$ & 24383,96 & 18287,97 & --- & 6095,99 \\
$\mathrm{NH}_{3}$ & --- & 517,54 & --- & --- \\
$\mathrm{CH}_{1,8} \mathrm{O}_{0,5} \mathrm{~N}_{0,2}$ & 10,85 & --- & 3750,86 & 3761,71 \\
$\mathrm{CO}_{2}$ & --- & --- & 7393,82 & 7393,82 \\
$\mathrm{H}_{2} \mathrm{O}$ & --- & --- & 272,49 & 272,49 \\
$\mathrm{C}_{2} \mathrm{H}_{6} \mathrm{O}$ & --- & --- & 7388,34 & 7388,34 \\
\hline
\end{tabular}

Tabla 7.

Balance de masa en fermentación acética, en Kg

\begin{tabular}{|lcccc|}
\hline Componente & Ingresa & Consume & Forma & Sale \\
\hline \hline $\mathrm{C}_{2} \mathrm{H}_{6} \mathrm{O}$ & 7388,34 & 1189,52 & --- & 6198,81 \\
$\mathrm{O}_{2}$ & 1616,26 & 1469,33 & --- & 146,92 \\
$\mathrm{~N}_{2}$ & 5320,45 & --- & --- & 5320,45 \\
$\mathrm{NH}_{3}$ & --- & 4,26 & --- & --- \\
$\mathrm{CH}_{1.4} \mathrm{O}_{0.4} \mathrm{~N}_{0.2}$ & 1,72 & --- & 28,60 & 30,32 \\
$\mathrm{CO}_{2}$ & --- & --- & 597,27 & 597,27 \\
$\mathrm{H}_{2} \mathrm{O}$ & --- & --- & 747,84 & 747,84 \\
$\mathrm{C}_{2} \mathrm{H}_{4} \mathrm{O}_{2}$ & --- & --- & 1254,77 & 1254,77 \\
\hline
\end{tabular}


En lo que respecta a los requerimientos energéticos se obtuvieron los siguientes resultados: en la fermentación alcohólica se requieren $2755,90 \mathrm{~kW}$, mientras que en la fermentación acética 4561,89 $\mathrm{kW}$.

La figura 5 muestra un diseño de reactor de lote, con los parámetros considerados en el diseño de reactores en función de las dimensiones y necesidades operativas del proceso de fermentación.

Tabla 8.

Factores de diseño para los reactores

\begin{tabular}{|lcc|}
\hline Factor & Alcohólico & Acético \\
\hline \hline Volumen reacción & $1,71 \mathrm{~m}^{3}$ & $1,24 \mathrm{~m}^{3}$ \\
Volumen efectivo & $2,14 \mathrm{~m}^{3}$ & $1,56 \mathrm{~m}^{3}$ \\
Área de la base $\left(A_{b}\right)$ & $1,7 \mathrm{~m}^{2}$ & $0,62 \mathrm{~m}^{2}$ \\
Diámetro del tanque $\left(\boldsymbol{D}_{\boldsymbol{T}}\right)$ & $1,5 \mathrm{~m}$ & $1,34 \mathrm{~m}$ \\
Diámetro del impelente $\left(\boldsymbol{D}_{\boldsymbol{i}}\right)$ & $0,49 \mathrm{~m}$ & $0,44 \mathrm{~m}$ \\
Número de Reynolds $\left(\boldsymbol{R}_{\boldsymbol{e} i}\right)$ & Turbulento & Turbulento \\
Potencia del agitado $(\boldsymbol{P})$ & $1,24 \mathrm{~W}$ & $0,58 \mathrm{~W}$ \\
Tiempo de mezcla $(\boldsymbol{t m})$ & $14,64 \mathrm{~S}$ & $15,26 \mathrm{~S}$ \\
\hline
\end{tabular}

El rendimiento obtenido en el producción alcohólica obtenidos con proceso de fermentación alcohólica fue del $75 \%$; de acuerdo con Lopez (2000), los rendimientos de

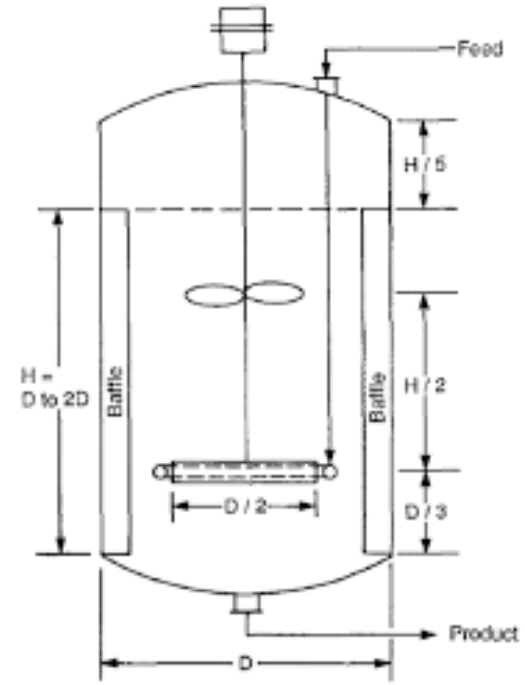

Figura 5. Diseño del reactor A continuación, en la tabla 8 se muestran los factores de diseño para los reactores de fermentación. 
levaduras modificadas y $70 \%$ para la levadura de pan.

Según Chauvet (1974), el vino se ha transformado en vinagre cuando su contenido de alcohol se ha reducido a $1^{\circ} \mathrm{GL}$ o $0,8 \mathrm{~g} \cdot \mathrm{mL}^{-1}$; sin embargo, al evaluar el vinagre obtenido se constató que poseía un grado alcohólico superior, lo que indica que el proceso fermentativo aún puede llevarse a cabo. En la fermentación alcohólica se obtuvo una conversión del $16,1 \%$ y se consume el $75,8 \%$ del oxígeno teórico. El bajo rendimiento obtenido se puede atribuir a la evaporación de los volátiles durante la fermentación acética, esta es una de las mayores causas de reducción en el rendimiento; en los sistemas discontinuos es necesario suministrar una cantidad considerable de aire, lo que trae consigo un aumento de las pérdidas por evaporación, mientras que a bajos caudales de aire, los rendimientos aumentan (Rubio et al., 2000). Ambos procesos fermentativos son endotérmicos; sin embargo, la fermentación acética requiere un 60,4 \% más de energía.

El requerimiento en volumen es mayor para la fermentación alcohólica.

E impelente seleccionado es la turbina Rushton. El tiempo de mezclado para la fermentación acética y alcohólica es $14,64 S$ y $15,26 \mathrm{~S}$ respectivamente. El control y diseño de biorreactores posee un permanente desarrollo en investigaciones y se requiere tiempo antes que un esquema de control bien desarrollado pueda ser implementado en biorreactores prácticos con alto desempeño (Echeverry et al., 2004).

\section{Conclusiones}

La implementación de la doble fermentación fue clave para la formación del sustrato secundario como era el vino que a su vez forma al ácido acético; sin embargo, los rendimientos obtenidos para los procesos fermentativos bifásicos fueron 75 y 16,1\% respectivamente. Para aumentar el rendimiento en la fermentación acética se pueden emplear mayores velocidades de agitación y mantener la temperatura al nivel mínimo aceptable para el proceso a los fines de minimizar las pérdidas por evaporación. El escalado del proceso se realizó en función de la demanda potencial del consumo de vinagre, en vista de los resultados obtenidos es necesaria la 
implementación de biorreactores en serie como criterio que garantice el mejor desempeño del proceso productivo para la obtención de vinagre.

\section{Agradecimientos}

Los integrantes del presente artículo agradecemos de manera muy cordial al Lic. Oswaldo García -Técnico del laboratorio de química y al Ing. Ernesto Rosero, PhD - catedrático de la materia de Ingeniería de los Bioprocesos de la carrera de Ingeniería Química de la UTM.

\section{Bibliografía}

Arnáiz, C., Isac, L., \& Lebrato, J. (2000). Determinación de la biomasa en procesos biológicos. Grupo de Tratamiento de Aguas Residuales, 45-52.

Barre, S., \& Stalin, M. (2003). Estudio nutricional comparativo de bokashis inoculados con levaduras y con microorganismos eficientes (EM) de primera y segunda generación a dos diferentes concentraciones en Zamorano.

Blanco, A., Quicazán, M., \& Cuenca, M. (2012). Efecto de algunas fuentes de nitrógeno en la fermentación alcohólica de miel. Vitae, 19(1), S234-S236.
Chauvet, M. (1974). Manual de viticultura. Madrid: Mundiprensa.

Corporación Financiera Nacional. CFN. (Septiembre de 2017). Obtenido de https://www.cfn.fin.ec/wpcont ent/uploads/2017/09/FchaSectorial-Banano.pdf.

Echeverry, N., Quintero, O., Ramírez, M., \& Álvarez, $\mathrm{H}$. (2004). Control de un bioreactor para fermentación alcohólica en continuo. CLCA'04.

Escalante, W. (2011). Actividad fermentativa de saccharomycodes ludwigii $\mathrm{y}$ evaluación de la síntesis de compuestos de importancia sensorial durante la fermentación de jugo de manzana. TIP Revista Especializada en Ciencias Químico-Biológicas, 12-23.

Ferreyra, M., \& Schvab, M. (2014). Obtención de vinagre de naranja en proceso semicontinuo, a escala laboratorio. Ciencia, Docencia y Tecnología, 154-165.

Hurtado, J. (2011). Correlación diagnóstica entre Tinción de Gram y Urocultivo en el diagnóstico de infección de vías urinarias. Universidad Católica Santiago de Guayaquil, 1-82.

ICBF. (2005). Tabla de Composición de Alimentos. Alimento: 


Banano común (musa azúcar. Agrociencia, 45(6),
sapientum).

INEN. (2012). NTE INEN 0341: Bebidas alcohólicas. Determinación de la acidez. INEN 0341, 1-14

INEN. (2013). Bebidas alcohólicas. Determinación del grado alcohólico en vinos. NTE INEN 0360, 1-15.

Llaguno, C., \& Polo, M. (1991). El vinagre de vino. España: Editorial CSIC - CSIC Press.

Lopez, M. (2000). Natural Antimicrobials from plants. En minimally processed fruits vegetables. Fundamentals and applications. Science, 235.

Mallouchos. (2003). Wine fermentations by immobilized and free cells at different temperatures. Effect of immobilization and temperature on volatile byproducts. Food Chemistry, 109-113

Mesas, M., \& Alegre, T. (1999). El papel de los microorganismos en la elaboración del vino. Journal of Food, 174-183.

Peláez-Acero, A., Meneses-Mayo, M., Miranda-Romero, L. A., Ayala-Martínez, M., CrosbyGalván, M. M., Loera-Corral, O., \& Megías-Rivas, M. D. (2011). Enzimas fibrolíticas producidas por fermentación en estado sólido para mejorar los ensilajes de caña de
Querol, A., Fernández-Espinar, M., del Olmo, M. \& Barrio, E. (2003). Evolución adaptativa de la levadura del vino. Revista internacional de microbiología de alimentos, 86(1-2), 3-10.

Romero, J. (2002). Efecto antagónico de $\mathrm{C}$. rugosa sobre microorganismos contaminantes de la uchuva nativa (Physalis peruviana). Microbiología industrial. Pontificia Universidad Javeriana. Facultad de Ciencias. Bogotá. 88 p.

Rubio, H., Salvador, M., \& Fregapane, G. (2000). Contribución a la mejora del proceso de acetificación para la producción industrial de vinagre de vino. Alimentación, equipos y tecnología, 209214.

Sellmer-Wilsberg, S. (2016). Proceedings of the Second Symposium on $\mathrm{R}+\mathrm{D}+\mathrm{l}$ for Vinegar. New strategies in process control for the production ofwine vinegar. Science, 127-134.

Tesfaye. (2002). Estudio de un sistema de fermentación sólida con agitación en la biotransformación del bagazo de caña de azúcar por la cepa Trichoderma viride M5-2. Revista Cubana de Ciencia Agrícola, 265-270. 
Yank, Martina, \& Corace. (2015).

Determinación de $\mathrm{CO}_{2}$ en

diferentes muestras de gas

mediante el uso del aparato

de Orsat. Energías

Renovables, 1-3. 